PROCEEDINGS OF THE

AMERICAN MATHEMATICAL SOCIETY

Volume 137, Number 1, January 2009, Pages 91-98

S 0002-9939(08)09545-2

Article electronically published on August 13, 2008

\title{
BASE LOCI OF LINEAR SYSTEMS AND THE WARING PROBLEM
}

\author{
MASSIMILIANO MELLA \\ (Communicated by Ted Chinburg)
}

\begin{abstract}
The Waring problem for homogeneous forms asks for additive decompositions of a homogeneous form $f$ into powers of linear forms. A classical problem is to determine when such a decomposition is unique. In this paper I refine my earlier work (Trans. Amer. Math. Soc. 358 (2006), 5523-5538) and answer this question under a divisibility assumption. To do this I translate the algebraic statement into a geometric one concerning the base loci of linear systems of $\mathbb{P}^{n}$ with assigned singularities.
\end{abstract}

\section{INTRODUCTION}

The Waring problem for forms is the quest for an additive decomposition of homogeneous polynomials into powers of linear ones. The subject has been widely considered in old times, $[\mathrm{Sy},[\mathrm{Hi},[\mathrm{Ri}]$ and $[\mathrm{Pa}$, with special regards to the existence of a unique decomposition of this type. The following, see RS, was the state of the art at the beginning of the $\mathrm{XX}^{\text {th }}$ century. A general form $f$ of degree $d$ in $n+1$ variables has a unique presentation as a sum of $s$ powers of linear forms in the following cases:

- $n=1, d=2 k-1$ and $s=k,[\mathrm{Sy}$;

- $n=3, d=3$ and $s=5$ Sylvester's Pentahedral Theorem [Sy];

- $n=2, d=5$ and $s=7$ [Hi], $\mathrm{Ri}$, $\mathrm{Pa}$.

After these remarkable results the Waring problem had kind of a rest and only quite recently come back on the main scene; see Ci for a complete account of all contributions ranging from algebraic geometry to commutative algebra and computer science.

I do expect that the ones listed are the only possible cases in which the decomposition is unique. A partial result in this direction is the following.

Theorem 1 ([Me $)$. Let $f$ be a general homogeneous form of degree d in $n+1$ variables. Assume that $d>n>1$. Then $f$ is expressible as a sum of $d$ th powers of linear forms in a unique way if and only if $n=2$ and $d=5$.

The aim of this paper is to give more evidence to the above expectation.

Received by the editors October 24, 2007, and, in revised form, January 8, 2008.

2000 Mathematics Subject Classification. Primary 14J70; Secondary 14N05, 14E05.

Key words and phrases. Waring, linear system, singularities, birational maps.

The author was partially supported by Progetto PRIN 2006 "Geometria sulle varietà algebriche" MUR. 
Theorem 2. Let $f$ be a general homogeneous form of degree $d$ in $n+1$ variables. Assume that $d \geq 5, n \geq 3$, and $\frac{\left(\begin{array}{c}n+d \\ n-1\end{array}\right)}{n}$ is an integer. Then $f$ is never expressible as a sum of dth powers of linear forms in a unique way.

Let me briefly comment upon the two theorems. The idea is to translate the original statement into one on birational maps of $\mathbb{P}^{n}$. To prove Theorem 1 it is then enough to study the singularities of special linear systems $\mathcal{G}_{d, n, l}$, with prescribed double points. This is done by a degeneration argument essentially borrowed from $[\mathrm{AH}$. To go beyond, namely $d \leq n$, one has to study the base locus of these special linear systems. The main difficulty is that the degeneration technique seems to be hopeless in this realm. To study the base locus of a linear system of projective dimension $n$ on an $n$-fold, one has to keep track of all the elements of a base, while, in general, the degeneration technique allows you to better understand a sublinear system, discarding completely the other elements. Instead of trying to determine the base locus of $\mathcal{G}_{d, n, l}$, I prove a weaker statement on the base locus on an open Zariski set containing the imposed points. This, under the divisibility assumption, is then enough to conclude.

The paper is organised as follows. I first introduce the main notation and preliminaries. Then I study the base locus of these special linear systems and bound the degree of the maps to prove Theorem 2, I would like to thank the referee for a careful reading of the manuscript.

\section{NOTATION AND PRELIMINARIES}

Unless otherwise stated I work over the field of complex numbers. First I introduce what is needed to study linear systems with prescribed singularities.

Definition 1.1. Let $p \in \mathbb{P}^{n}$ be a point. The double point at $p$ in $\mathbb{P}^{n}$ is the scheme given by the square of the ideal sheaf of $p$. If $P \subset \mathbb{P}^{n}$ is a collection of points, I denote by $P^{2}$ the double points supported on $P$. In particular the linear system $\left|\mathcal{I}_{P^{2}}(d)\right|$ is given by hypersurfaces of degree $d$ singular at $P$.

Given a collection of points $X=Q \cup Q_{H}$, with $Q_{H}$ supported on a hyperplane $H$, let $\tilde{X}$ be the residual of $X^{2}$ with respect to $H$. That is, $\tilde{X}=Q^{2} \cup Q_{H}$. Then there is the Castelnuovo exact sequence given by

$$
0 \rightarrow \mathcal{I}_{\tilde{X}}(d-1) \rightarrow \mathcal{I}_{X^{2}}(d) \rightarrow \mathcal{I}_{Q^{2}, H}(d) \rightarrow 0 .
$$

This gives the following sequence on cohomology:

$$
0 \rightarrow H^{0}\left(\mathbb{P}^{n}, \mathcal{I}_{\tilde{X}}(d-1)\right) \rightarrow H^{0}\left(\mathbb{P}^{n}, \mathcal{I}_{X^{2}}(d)\right) \rightarrow H^{0}\left(H, \mathcal{I}_{Q_{H}^{2}}(d)\right)
$$

Definition 1.2. Consider a collection $P$ of $l$ general points in $\mathbb{P}^{n}$. Define

$$
\mathcal{G}_{d, n, l}:=\left|\mathcal{I}_{P^{2}}(d)\right| .
$$

Fix a hyperplane $H$ and a collection $X=Q \cup Q_{H}$, where $Q$ is given by $(l-h)$ general points in $\mathbb{P}^{n}$, and $Q_{H}=\bigcup_{1}^{h} q_{j}$ is given by $h$ general points in $H$. Define

$$
\mathcal{H}_{H, d, n, l, h}:=\left|\mathcal{I}_{X^{2}}(d)\right| \text {. }
$$

In this paper I am interested in nonempty linear systems of type $\mathcal{G}_{d, n, l}$. For this I introduce the following definition. 
Definition 1.3. I say that the linear system $\mathcal{G}_{d, n, l}$ is expected if

$$
\operatorname{dim} \mathcal{G}_{d, n, l}=\left(\begin{array}{c}
n+d \\
n
\end{array}\right)-(n+1) l-1
$$

Moreover if $\mathcal{G}_{d, n, l}$ is expected and $\operatorname{dim} \mathcal{G}_{d, n, l} \geq 0$, I say that it is expected and effective.

Note that if $\mathcal{G}_{d, n, l}$ is expected and effective, then $\mathcal{G}_{d, n, l^{\prime}}$ is expected and effective for any $l^{\prime}<l$, and similarly for linear systems of type $\mathcal{H}$. I say that $\mathcal{H}_{H, d, n, l, h}$ is expected and effective if

$$
\operatorname{dim} \mathcal{H}_{H, d, n, l, h}=\left(\begin{array}{c}
n+d \\
n
\end{array}\right)-(n+1) l-1 \geq 0 .
$$

Note that if $\mathcal{H}_{H, d, n, l, h}$ is expected and effective, then by semi-continuity, $\mathcal{G}_{d, n, l}$ is expected and effective. In the following I frequently ask expected and effective linear systems of type $\mathcal{H}$ to satisfy the following further properties. The linear system $\mathcal{H}_{H, d, n, l, h}$ is what I need (wIn) if

- $\mathcal{H}_{H, d, n, l, h}$ is expected and effective,

- $\left|\mathcal{H}_{H, d, n, l, h} \otimes \mathcal{I}_{H}\right| \neq \emptyset$,

- (1) is a short exact sequence.

I am interested in studying the base loci of linear systems of type $\mathcal{G}_{d, n, l}$. To do this I use a degeneration argument.

Lemma 1.4 ([Me, Lemma 1.5]). Let $\Delta$ be a complex disk around the origin. Consider the product $V=\mathbb{P}^{n} \times \Delta$, with the natural projections, $\pi_{1}$ and $\pi_{2}$. Let $V_{t}=\mathbb{P}^{n} \times\{t\}$ and $\mathcal{O}_{V}(d)=\pi_{1}^{*}\left(\mathcal{O}_{\mathbb{P}^{n}}(d)\right)$. Fix a configuration $q_{1}, \ldots, q_{l}$ of $l$ points on $V_{0}$ and let $\sigma_{i}: \Delta \rightarrow V$ be sections such that $\sigma_{i}(0)=q_{i}$ and $\left\{\sigma_{i}(t)\right\}_{i=1, \ldots, l}$ are general points of $V_{t}$ for $t \neq 0$. Let $P_{t}=\bigcup_{i=1}^{l} \sigma_{i}(t)$.

Consider the linear system $\mathcal{H}_{t}=\left|\mathcal{O}_{V_{t}}(d) \otimes \mathcal{I}_{P_{t}^{2}}\right|$. Assume that $l<\left\lceil\frac{\left(\begin{array}{c}d+n \\ n\end{array}\right)}{n+1}\right\rceil$ and $\operatorname{dim} \mathcal{H}_{0}=\operatorname{dim} \mathcal{H}_{t}$, for $t \in \Delta$. Let $\psi_{i}(t):=\operatorname{dim}_{\sigma_{i}(t)}$ Bs $\mathcal{H}_{t}$. Then for $t \neq 0$, we have

$$
\psi_{i}(t) \leq \min \left\{j \mid \psi_{j}(0)\right\} .
$$

The way to pass from linear systems of type $\mathcal{H}$ to those of type $\mathcal{G}$ is the content of the following.

Corollary 1.5. Assume that $\mathcal{H}_{H, d, n, l, h}$ is wIn and $\mathrm{Bs} \mathcal{H}_{H, d, n, l, h}=q_{i}^{2}$ in a neighbourhood of a point $q_{i}$. Then $\operatorname{Bs}\left(\mathcal{G}_{d, n, l}\right)=P^{2}$ in a neighbourhood of $P$.

Proof. I am in the condition to apply Lemma 1.4 thus $\psi_{i}(t)=0$ for any $i$. Moreover the blowup of $q_{i}$ solves $\mathrm{Bs} \mathcal{H}_{H, d, n, l, h}$ in a neighbourhood of $q_{i}$. This means that, with the lemma's notation, the blowup of $\sigma_{i}(\Delta)$ resolves Bs $\mathcal{G}_{d, n, l}$ in a neighbourhood of $p_{i}$. A monodromy argument shows that this is true for any point $p_{i} \in P$.

I recall a way to translate the existence of a unique decomposition into a statement on the map defined by linear systems of type $\mathcal{G}_{d, n, k}$.

Proposition 1.6 ([Me pg. 5529]). Let $f$ be a general homogeneous form of degree $d$ in $n+1$ variables, with $n \geq 2$. Then $f$ is expressible as a sum of $(k+1)$ powers of linear forms in a unique way only if the map associated to the linear system $\mathcal{G}_{d, n, k}$ is birational and $k=\frac{\left(\begin{array}{c}d+n \\ n\end{array}\right)}{n+1}-1$ is an integer. 
To test this condition, when $d \leq n$, I have to understand the base locus of the linear system $\mathcal{G}_{d, n, k}$. In the following I shall do this by a degeneration argument. For this let me recall the following result.

Lemma 1.7 ([Me, Lemma 3.4]). Fix a hyperplane $H \subset \mathbb{P}^{n}$. Let

and

$$
l_{d}:=l=\left\lceil\frac{\left(\begin{array}{c}
n+d+1 \\
n
\end{array}\right)}{n+1}\right\rceil-\left\lceil\frac{\left(\begin{array}{c}
n+d \\
n-1
\end{array}\right)}{n}\right\rceil
$$

$$
h_{d}:=h=\left\lceil\frac{\left(\begin{array}{c}
n+d+1 \\
n
\end{array}\right)}{n+1}\right\rceil-\left\lceil\frac{\left(\begin{array}{c}
n+d \\
n-1
\end{array}\right)}{n}\right\rceil-\left\lceil\frac{\left(\begin{array}{c}
n+d \\
n
\end{array}\right)}{n+1}\right\rceil+\left\lceil\frac{\left(\begin{array}{c}
n-1+d \\
n-1
\end{array}\right)}{n}\right\rceil .
$$

Assume that $d \geq 4$ and $n \geq 3$. Then $\mathcal{H}_{H, d, n, l, h}$ is wIn.

\section{BASE LOCI OF LINEAR SYSTEMS}

First I study the base locus of $\mathcal{G}_{d, n, l}$ when $l$ is quite small.

Theorem 2.1. Assume that $d \geq 4, n \geq 3$, and $l \leq\left\lceil\frac{\left(\begin{array}{c}n+d+1 \\ n\end{array}\right)}{n+1}\right\rceil-\left\lceil\frac{\left(\begin{array}{c}n+d \\ n-1\end{array}\right)}{n}\right\rceil$. Then $\mathcal{G}_{d, n, l}$ is expected and effective and $\operatorname{Bs}\left(\mathcal{G}_{d, n, l}\right)=P^{2}$ in a neighbourhood of $P$.

Proof. It is clear that it is enough to prove the statement for $l=\left\lceil\frac{\left(\begin{array}{c}n+d+1 \\ n\end{array}\right)}{n+1}\right\rceil-\left\lceil\frac{\left(\begin{array}{c}n+d \\ n-1\end{array}\right)}{n}\right\rceil$. I prove the claim by induction on $n$.

Let $l^{\prime}=\left\lceil\frac{\left(\begin{array}{c}n+d \\ n\end{array}\right)}{n+1}\right\rceil-\left\lceil\frac{\left(\begin{array}{c}n+d-1 \\ n-1\end{array}\right)}{n}\right\rceil$ and $h=l-l^{\prime}$. To be able to apply induction on $n$ I need to prove that $\gamma=h-\left(\left\lceil\frac{\left(\begin{array}{c}n+d \\ n-1\end{array}\right)}{n}\right\rceil-\left\lceil\frac{\left(\begin{array}{c}n-1+d \\ n-2\end{array}\right)}{n-1}\right\rceil\right) \leq 0$. Note that

$$
h=\left\lceil\frac{\left(\begin{array}{c}
n+d+1 \\
n
\end{array}\right)}{n+1}\right\rceil-\left\lceil\frac{\left(\begin{array}{c}
n+d \\
n-1
\end{array}\right)}{n}\right\rceil-\left\lceil\frac{\left(\begin{array}{c}
n+d \\
n
\end{array}\right)}{n+1}\right\rceil+\left\lceil\frac{\left(\begin{array}{c}
n+d-1 \\
n-1
\end{array}\right)}{n}\right\rceil<\frac{\left(\begin{array}{c}
n-1+d \\
n-1
\end{array}\right)}{n}-\frac{\left(\begin{array}{l}
n+d \\
n-1
\end{array}\right)}{n(n+1)}+2
$$

so that

$$
\begin{aligned}
\gamma=h-\left(\left\lceil\frac{\left(\begin{array}{c}
n+d \\
n-1
\end{array}\right)}{n}\right\rceil-\left\lceil\frac{\left(\begin{array}{c}
n-1+d \\
n-2
\end{array}\right)}{n-1}\right\rceil\right) & <\frac{\left(\begin{array}{c}
n+d-1 \\
n-1
\end{array}\right)}{n}+\frac{\left(\begin{array}{c}
n+d-1 \\
n-2
\end{array}\right)}{n-1}-\left(\begin{array}{c}
n+d \\
n-1
\end{array}\right) \frac{n+2}{n(n+1)}+3 \\
< & \frac{(n+d-1) \cdot \ldots \cdot(d+2)}{n !}\left(1-\frac{n+d}{n+1}\right)+3=\alpha .
\end{aligned}
$$

Standard computations give $\alpha \leq 0$ in the following cases:

$$
\begin{aligned}
& \text {. } d \geq 4 \text { and } n \geq 9, \\
& \text {. } d \geq 5 \text { and } n \geq 6, \\
& \text {. } d \geq 6 \text { and } n \geq 4, \\
& \text {. } d \geq 9 \text { and } n \geq 3 .
\end{aligned}
$$

In the remaining cases I have to compute $\gamma$ directly:

\begin{tabular}{|c|cccccc|}
\hline$(d, n)$ & $(4,3)$ & $(4,4)$ & $(4,5)$ & $(4,6)$ & $(4,7)$ & $(4,8)$ \\
\hline$\gamma$ & -1 & 0 & -3 & -1 & -3 & -2 \\
\hline$(d, n)$ & $(5,3)$ & $(5,4)$ & $(5,5)$ & $(6,3)$ & $(7,3)$ & $(8,3)$ \\
\hline$\gamma$ & -2 & -2 & -2 & -1 & -1 & -5 \\
\hline
\end{tabular}

This shows that I can apply the induction hypothesis on $\mathcal{G}_{d, n-1, h}$. Fix a general hyperplane $H \subset \mathbb{P}^{n}$. Then by Lemma 1.7 the linear system $\mathcal{H}_{H, d, n, l, h}$ is wIn.

Let $Q$ be a collection of $l-h$ general points in $\mathbb{P}^{n}$. The main result in $[\mathrm{AH}$ ] reads that the linear systems $\mathcal{G}_{d-1, n, l-h}$ and $\mathcal{G}_{d-1, n, l-h+1}$ are both expected and effective. 
The latter ensures that the morphism associated to the sections of $\mathcal{G}_{d-1, n, l-h}$ is generically finite and therefore the general intersection of $D_{1}, \ldots, D_{n-1} \in \mathcal{G}_{d-1, n, l-h}$ contains an irreducible curve.

Let $H$ be a hyperplane and $C \subset D_{1} \cap \ldots \cap D_{n-1}$ be a general one dimensional irreducible component. The linear system $\mathcal{H}_{H, d, n, l, h}$ is wIn. An easy computation shows that $\operatorname{dim} \mathcal{G}_{d-1, n, l-h} \sim h+\frac{\left(\begin{array}{c}n+d \\ n-1\end{array}\right)}{n}$. Let $Q_{H}$ be a general set of $h$ distinct points in $H$. Then there are $n-1$ general elements $D_{1}, \ldots, D_{n-1} \in \mathcal{G}_{d-1, n, l-h}$ containing $Q_{H}$, and I can therefore choose the hyperplane $H$ in such a way that

. $X=Q \cup Q_{H}$ and $Q_{H} \subset C \cap H$,

. $H$ is tangent to $C$ at a point, say $q_{1} \in Q_{H}$.

Let $\epsilon: Y \rightarrow \mathbb{P}^{n}$ be the blowup of $q_{1}$ with exceptional divisor $E, H_{Y}=\epsilon_{*}^{-1} H$, and $\mathcal{H}_{Y}=\epsilon_{*}^{-1} \mathcal{H}_{H, d, n, l, h}$. The choice of $H$ yields

$$
\text { Bs } \mathcal{H}_{Y} \cap E \subset H_{Y} \cap E \text {. }
$$

The linear system $\mathcal{H}_{H, d, n, l, h}$ is $\mathbf{w I n}$; in particular all elements of $\mathcal{G}_{d, n-1, h}$ lift to elements in $\mathcal{H}_{H, d, n, l, h}$. By the induction hypotheses the linear system $\mathcal{G}_{d, n-1, h}$ has the correct base locus in a neighbourhood of $q_{1}$. Then $\operatorname{Bs}\left(\mathcal{H}_{H, d, n, l, h}\right)=q_{1}^{2}$ in a neighbourhood of $q_{1}$. I will then conclude the proof by Corollary 1.5 .

It remains to prove the first step of the induction. That is the statement for $n=3$. Arguing as in the induction step one can prove that $\mathcal{G}_{d, 2, h}$ has the expected base locus. This is a well-known result, but I have no correct reference for it. The special case I need is particularly simple, and one can prove it in a few lines. The hypotheses give

$$
h<\frac{d+2}{3}+\frac{3}{2}<\left(\begin{array}{c}
\left\lfloor\frac{d}{2}\right\rfloor+2 \\
2
\end{array}\right)-1 .
$$

Then there are reducible divisors $D=D_{1} \cup D_{2} \in \mathcal{G}_{d, 2, l}$ such that $P \in D_{i}$. This means that the base locus in a neighbourhood of $P$ is the one prescribed.

Remark 2.2. Note that an important feature of the above proof is that the linear system $\mathcal{H}_{H, d, n, l, h}$ breaks into two parts, $\mathcal{G}_{d-1, n, l-h}$ and $\mathcal{G}_{d, n-1, h}$, both of dimension at least $n+1$. This is of course not true when one imposes the maximal number of double points. The divisibility condition in Theorem 2 forces all the linear systems "on one side".

Theorem 2.3. Assume that $d+1 \geq 5, l=\frac{\left(\begin{array}{c}n+d+1 \\ n\end{array}\right)}{n+1}-1$ and $\frac{\left(\begin{array}{c}n+d \\ n-1\end{array}\right)}{n}$ are integers. Then $\operatorname{Bs}\left(\mathcal{G}_{d+1, n, l}\right)=P^{2}$ in a neighbourhood of $P$. Assume that $n \geq 3$. Then the map given by the linear system $\mathcal{G}_{d+1, n, l}$ is not birational.

Proof. Let $h=\frac{\left(\begin{array}{c}n+d \\ n-1\end{array}\right)}{n}$ be an integer. The linear system $\mathcal{H}_{H, d+1, n, l, h}$ is wIn by [Me, Proof of Theorem 4.3]. The numerical hypothesis yields $\operatorname{dim} \mathcal{G}_{d, n, l-h}=n+h$ and $\operatorname{dim} \mathcal{H}_{H, d+1, n, l, h}=n$. In particular all elements of $\mathcal{H}_{H, d+1, n, l, h}$ contain the hyperplane $H$. Let $P$ be a length $l-h 0$-scheme and $X=P \cup Q_{H}$ a length $l$ 0 -scheme.

The main result in $\left[\mathrm{AH}\right.$ ] reads that the linear systems $\mathcal{G}_{d, n, l-h}$ and $\mathcal{G}_{d, n, l-h+1}$ are both expected and effective. The latter ensures that the morphism associated to the sections of $\mathcal{G}_{d, n, l-h}$ is generically finite. By Theorem 2.1, $\operatorname{Bs}\left(\mathcal{G}_{d, n, l-h}\right)=P^{2}$ in a neighborhood of $P$, and therefore the general intersection of $D_{1}, \ldots, D_{n-1} \in$ $\mathcal{G}_{d, n, l-h}$ contains an irreducible curve passing through $P$. 
Let $D_{1}, \ldots D_{n-1} \in \mathcal{G}_{d, n, l-h}$ be general elements and $C=D_{1} \cap \ldots \cap D_{n-1}$ the irreducible curve passing through $P$. Let $H$ be a hyperplane and $Q_{H}$ a general set of $h$ distinct points in $H$. Then there are $n-1$ general elements $D_{1}, \ldots, D_{n-1} \in$ $\mathcal{G}_{d-1, n, l-h}$ containing $Q_{H}$, and I can therefore choose the hyperplane $H$ in such a way that $Q_{H} \subset C \cap H$. The general choice of $H$ allows me to assume that the general element in $\left|\mathcal{G}_{d, n, l-h} \otimes \mathcal{I}_{Q_{H}}\right|$ is not tangent to $C$ at $p_{1}$. This shows that $\operatorname{Bs}\left(\mathcal{H}_{H, d+1, n, l, h}\right)=p_{1}^{2}$ in a neighbourhood of $p_{1}$. Then Corollary [1.5 yields $\operatorname{Bs}\left(\mathcal{G}_{d+1, n, l}\right)=P^{2}$ in a neighbourhood of $P$.

Let $\chi: \mathbb{P}^{n} \rightarrow \mathbb{P}^{n}$ be the map given by the linear system $\mathcal{H}_{H, d+1, n, l, h}$ or equivalently $\left|\mathcal{G}_{d, n, l-h} \otimes \mathcal{I}_{Q_{H}}\right|$. I will decompose $\chi$ into several steps. Let $\epsilon: Z \rightarrow \mathbb{P}^{n}$ be the resolution of $\operatorname{Bs} \mathcal{G}_{d, n, l-h}$, with $\mathcal{G}_{Z}=\epsilon_{*}^{-1} \mathcal{G}_{d, n, l-h}$ and $H_{Z}=\epsilon_{*}^{-1} H$. I have the following:

- $E_{1}, \ldots, E_{l-h}$, the exceptional divisors over $P$, are all projective spaces of dimension $n-1$,

. the curve $C_{Z}=\epsilon_{*}^{-1} C$ is a curve section of $\mathcal{G}_{Z}$.

Let $\Psi: Z \rightarrow \mathbb{P}^{n+h}$ be the morphism associated to the linear system $\mathcal{G}_{Z}$ :

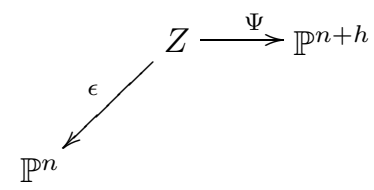

Then $\Psi\left(E_{i}\right)$ is a, eventually projected, 2-Veronese embedding of $\mathbb{P}^{n-1}$. This bounds the dimension of the span of $\Psi\left(E_{i}\right)$ :

$$
\operatorname{dim}\left\langle\Psi\left(E_{i}\right)\right\rangle<\left(\begin{array}{c}
n+1 \\
2
\end{array}\right)
$$

in particular $\Psi\left(E_{i}\right)$ is always degenerate. I already proved that $\operatorname{Bs}\left(\mathcal{G}_{d, n, l-h}\right)=p_{1}^{2}$ in a neighbourhood of $p_{1}$. Then I have

$$
\#\left(\Psi\left(C_{Z} \cap E_{1}\right)\right)=\mathcal{G}_{Z}^{n-1} \cdot E_{1}=2^{n-1}
$$

and by construction $\left\langle\Psi\left(C_{Z}\right)\right\rangle=\mathbb{P}^{h+1}$.

To complete the map $\chi$, I have to project from a linear space $\Lambda \cong \mathbb{P}^{h-1}$ the $h$-secant to $\Psi\left(H_{Z}\right)$.

Assume that the map $\chi$ is birational. Then the projection from $\Lambda$, say $\Pi_{\Lambda}$, is birational. Let $\Lambda=\left\langle\Lambda_{1}, x\right\rangle$ for some point $x$ and linear space $\Lambda_{1}$. Let me further factor this projection with the projection from $\Lambda_{1}$ and then from $x$ :

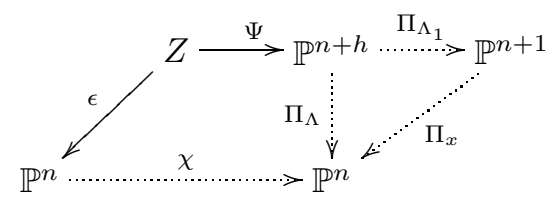

The divisor $\Pi_{\Lambda_{1}}(\Psi(Z))$ is a hypersurface of degree, say $j$. The projection $\Pi_{x}$ is birational only if

$$
\operatorname{mult}_{\Pi_{\Lambda_{1}}(x)} \Pi_{\Lambda_{1}}(\Psi(Z))=j-1
$$

On the other hand, $x$ is a general point of $\Psi\left(H_{Z}\right)$. Therefore this forces

$$
\operatorname{mult}_{\Pi_{\Lambda_{1}}\left(\Psi\left(H_{Z}\right)\right)} \Pi_{\Lambda_{1}}(\Psi(Z))=j-1 .
$$


Claim 1. $\Pi_{\Lambda_{1}}\left(\Psi\left(H_{Z}\right)\right)$ is a nondegenerate generically smooth codimension 2 subvariety of $\mathbb{P}^{n+1}$.

Proof of the claim. The variety $\Pi_{\Lambda_{1}}\left(\Psi\left(H_{Z}\right)\right)$ is degenerate only if there is an element in $\mathcal{G}_{d-1, n, l-h}$. To check this, by the main result in $[\mathrm{AH}$, it is enough to verify the following inequality:

$$
\left(\begin{array}{c}
n+d-1 \\
n
\end{array}\right)<\left(\begin{array}{c}
n+d+1 \\
n
\end{array}\right)-\left(\begin{array}{c}
n+d \\
n-1
\end{array}\right) \frac{n+1}{n}-(n+1) .
$$

Moreover $\Pi_{\Lambda_{1}} \Psi\left(H_{Z}\right)$ is a general projection from $h-1$ general points of $\Psi\left(H_{Z}\right) \subset$ $\mathbb{P}^{n+h}$. Therefore by the trisecant lemma (see for instance [CC, Proposition 2.6]) the map is birational. In particular I have $\operatorname{dim} \Pi_{\Lambda_{1}}\left(\Psi\left(H_{Z}\right)\right)=n-1$.

The claim says that the secant variety of $\Pi_{\Lambda_{1}}\left(\Psi\left(H_{Z}\right)\right)$ fills up $\mathbb{P}^{n+1}$. Therefore by equation (4) I have

$$
2(j-1) \leq j .
$$

That is, $j=2$ and $\Pi_{\Lambda_{1}}(\Psi(Z))$ is smooth along $\Pi_{\Lambda_{1}}\left(\Psi\left(H_{Z}\right)\right)$. The general choice of the points allows us to conclude that

$$
\operatorname{deg} \Psi(Z)=2+(h-1)=h+1 .
$$

To get a lower bound on the degree of $\Psi(Z)$, I use the degenerate variety $\Psi\left(E_{i}\right)$. Consider a general hyperplane

$$
A \subset\left\langle\Psi\left(C_{Z}\right)\right\rangle \cong \mathbb{P}^{h+1}
$$

containing $\Psi\left(E_{i}\right) \cap \Psi\left(C_{Z}\right)$. Then for such an $A$, by equations (2) and (3) I have

$$
A \cdot \Psi\left(C_{Z}\right) \geq 2^{n-1}+h+1-\left(\begin{array}{c}
n+1 \\
2
\end{array}\right) .
$$

A simple computation gives, for $n \geq 5$,

$$
2^{n-1}>\left(\begin{array}{c}
n+1 \\
2
\end{array}\right)
$$

Therefore for $n \geq 5$ I conclude

$$
\operatorname{deg} \Psi\left(C_{Z}\right)>h+1 .
$$

This proves that $\chi$ is finite and not birational. Then, by the usual degeneration argument, the map given by $\mathcal{G}_{d+1, n, l}$ is not birational.

Note that for $n=3,4$ the statement is a consequence of [Me, Theorem 1].

I am now able to prove the main theorem.

Proof of Theorem 2. The existence of a unique decomposition translates, by Proposition 1.6, in the fact that $\mathcal{G}_{d, n, l}$, with $l=\frac{\left({ }_{n}^{n+d+1}\right)}{n+1}-1$, gives a birational map to $\mathbb{P}^{n}$. For $n \leq 4$ the result is proved in $[\mathrm{Me}$. For $n \geq 5$, using Theorem 2.1] I conclude that such a linear system cannot exist.

Remark 2.4. If the map induced by sections of $\mathcal{G}_{d, n, l}$ is birational, then a general hypersurface of degree $d$, with $l$ ordinary nodes, [Me, is rational. This is quite against general expectations, at least for $d$ large enough. Unfortunately I do not know any direct method to prove the nonrationality of these special hypersurfaces. One should confront this with the Kollár result, [Ko], on nonrationality of very general smooth hypersurfaces of degree roughly bounded by $2 / 3$ the dimension. 


\section{REFERENCES}

[AH] J. Alexander, A. Hirschowitz, Polynomial interpolation in several variables, J. Algebraic Geom. 4 (1995), no. 2, 201-222. MR1311347 (96f:14065)

[AC] E. Arbarello, M. Cornalba, Footnotes to a paper of Beniamino Segre, Math. Ann. 256 (1981), no. 3, 341-362. MR626954 (83d:14016)

[CC] L. Chiantini, C. Ciliberto, Weakly defective varieties, Trans. Amer. Math. Soc. 354 (2002), no. 1, 151-178. MR1859030(2003b:14063)

[Ci] C. Ciliberto, Geometric aspects of polynomial interpolation in more variables and of Waring's problem, European Congress of Mathematics, Vol. I (Barcelona, 2000), 289-316, Progr. Math., 201, Birkhäuser, Basel, 2001. MR 1905326 (2003i:14058)

[Hi] D. Hilbert, Lettre adressée à M. Hermite, Gesam. Abh. vol II, 148-153.

[Ia] A. Iarrobino, Inverse system of a symbolic power. II. The Waring problem for forms, J. Algebra 174 (1995), no. 3, 1091-1110. MR1337187(96i:13018)

[Ko] J. Kollár, Nonrational hypersurfaces, J. Amer. Math. Soc. 8 (1995), no. 1, 241-249. MR 1273416 (95f:14025)

[Me] M. Mella, Singularities of linear systems and the Waring problem, Trans. Amer. Math. Soc. 358 (2006), no. 12, 5523-5538. MR2238925 (2007h:14059)

[Pa] F. Palatini, Sulla rappresentazione delle forme ternarie mediante la somma di potenze di forme lineari, Rom. Acc. L. Rend. 12 (1903) 378-384.

[RS] K. Ranestad, F. Schreyer, Varieties of sums of powers, J. Reine Angew. Math. 525 (2000), 147-181. MR1780430 (2001m:14009)

[Ri] H.W. Richmond, On canonical forms, Quart. J. Pure Appl. Math. 33 (1904) 967-984.

[Sy] J.J. Sylvester, Collected works, Cambridge University Press (1904).

Dipartimento di Matematica, Università di Ferrara, 44100 Ferrara, Italia

E-mail address: mll@unife.it 